\title{
Performance Evaluation of Arc-Electrodes Systems for High Temperature Materials Processing by Computational Simulation
}

\author{
Hideya NISHIYAMA, Takehiko SATO and Kazumune TAKAMURA ${ }^{1)}$ \\ Institute of Fluid Science, Tohoku University, Katahira, Aoba-ku, Sendai 980-8577 Japan. \\ 1) Matsushita Electric Industrial Co., Ltd., Kadoma 571-8501 Japan.
}

(Received on September 24, 2002; accepted in final form on February 4, 2003)

\begin{abstract}
Thermofluid analysis of an arc flow and the evaluation of the cathode lifetime have been conducted by a computational simulation for the performance improvement of arc-electrodes systems. The effects of arc current, electrode gap, inlet gas flow rate and cathode vertex angle on the temperature and velocity fields of an arc flow are clarified by parametric computations to optimize the operating conditions and torch geometry. The temperature field by an arc flow model shows the quantitative agreement with the available experimental data in the local thermodynamic equilibrium (LTE) region. The cathode lifetime is evaluated successfully by coupling with the computated thermofluid field of an arc flow. The effects of different operating conditions on the cathode lifetime are also discussed for longer lifetime of the arc-electrodes systems.
\end{abstract}

KEY WORDS: computational simulation; arc; electrode; interaction; lifetime evaluation.

\section{Introduction}

Thermal plasma flow can be regarded as a multi-functional fluid, since it has high energy flux, chemical reactivity, various gases selectivity and electromagnetic controllability. ${ }^{1)}$ Since the stable and controlled heating can be performed by an arc, it has been extensively utilized as a heat source for welding, cutting and furthermore in the waste treatment. ${ }^{2}$ ) However, the arc processing needs relatively higher cost compared with the conventional treatment by combustion, since the arc can be produced only by large electric power and also the expensive electrodes and reactor are easily consumed for short running time. ${ }^{3)}$ For this reason, the optimization of the operating conditions and torch design for the improvement of cost performance and for the extension of cathode lifetime has been expected eagerly in the industrial world. Nevertheless, it is experimentally difficult to clarify the physical phenomenon in detail and to optimize the operating conditions of an arc-electrodes system, since the thermofluid field of an arc is very complex in the extreme conditions. Therefore, the numerical modeling is expected to be one of the effective approach to solve this problem. $^{4-7)}$

In the present study the thermofluid analysis of free burning arc and tungsten cathode life evaluation ${ }^{8,9)}$ are conducted to provide the optimum operating conditions of an arc for the high energy efficiency and for a long life of electrode. In the thermofluid analysis, the electric field is solved in the total region including an arc flow zone and both cathode and anode regions by giving heat flux condition between arc and electrodes without assuming the distributions of temperature and electric current on the electrode surfaces. ${ }^{10)}$ The effects of arc current, inlet flow rate, electrode gap and cathode vertex angle on the thermofluid characteristics are clarified by computational simulation. Next, the numerical model is adopted in the evaluation of tungsten electrode life, considering the thorium diffusion inside the cathode and also thorium adsorption and desorption at the cathode tip. The time evolution of cathode surface temperature with the cathode vertex angle and applied arc current is evaluated by coupling with the cathode temperature distribution and electric current distribution previously obtained in the arc analysis. ${ }^{11)}$

\section{Arc Flow Analysis}

\subsection{Governing Equations}

Figure 1 shows a schematic illustration of a free burning arc-electrodes system and the coordinate system. In order to derive the governing equations, the following assumptions are introduced. ${ }^{4-7)}$

(1) The argon arc is regarded as an ideal gas and a continuous fluid. It is optically thin in Local Thermodynamic Equilibrium (LTE) state.

(2) The thermofluid field is laminar, steady and axisymmetrical two-dimensional (2D).

(3) The induction magnetic field has an axisymmetrical and azimuthal component.

(4) There are temperature dependent thermodynamic and transport properties.

(5) The gravitational force, viscous dissipation and compressed work are neglected.

(6) Only radiation loss from Ar is considered.

(7) Radiation, melting, evaporation and deformation on the both cathode and anode surfaces are neglected to avoid complexity. 


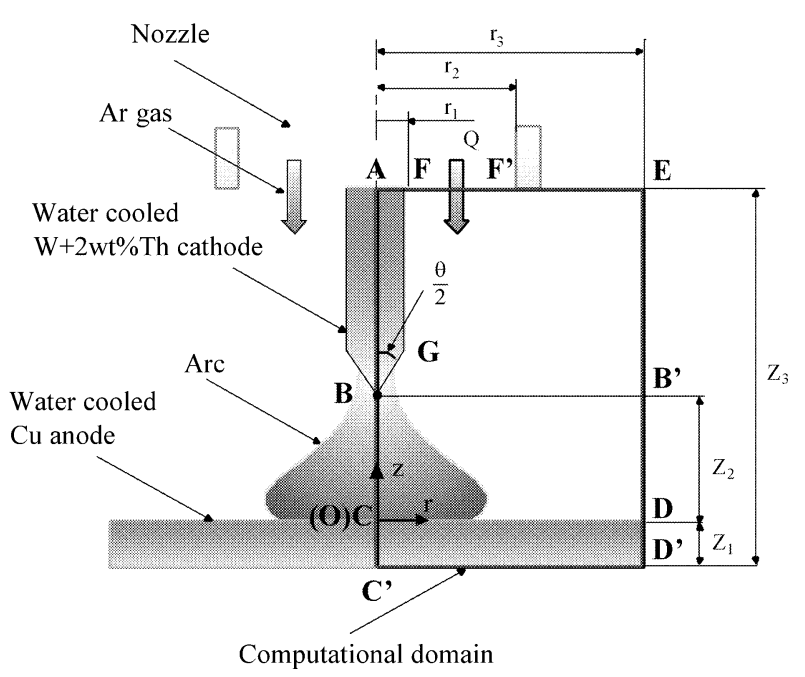

Fig. 1. Schematic illustration of an arc-electrodes system.

Under these assumptions, the governing equations for arc and electrodes regions are presented as follows:

Conservation of Mass

$$
\frac{\partial}{\partial z}(\rho u)+\frac{1}{r} \frac{\partial}{\partial r}(\rho r v)=0
$$

Conservation of Momentum

$$
\begin{aligned}
& \frac{\partial}{\partial z}(\rho u u)+\frac{1}{r} \frac{\partial}{\partial r}(\rho r u v) \\
& =-\frac{\partial p}{\partial z}+2 \frac{\partial}{\partial z}\left(\eta \frac{\partial u}{\partial z}\right)+\frac{1}{r} \frac{\partial}{\partial r}\left(\eta r\left(\frac{\partial u}{\partial r}+\frac{\partial v}{\partial z}\right)\right) \\
& -\frac{2}{3} \frac{\partial}{\partial z}\left(\eta\left(\frac{\partial u}{\partial z}+\frac{1}{r} \frac{\partial(r v)}{\partial r}\right)\right)+j_{r} B_{\theta}
\end{aligned}
$$

$$
\begin{aligned}
& \frac{\partial}{\partial z}(\rho v u)+\frac{1}{r} \frac{\partial}{\partial r}(\rho r v v) \\
& =-\frac{\partial p}{\partial r}+\frac{\partial}{\partial z}\left(\eta\left(\frac{\partial v}{\partial z}+\frac{\partial u}{\partial r}\right)\right)+\frac{2}{r} \frac{\partial}{\partial r}\left(\eta r \frac{\partial v}{\partial r}\right) \\
& \quad-\frac{2}{3} \frac{\partial}{\partial r}\left(\eta\left(\frac{\partial u}{\partial z}+\frac{1}{r} \frac{\partial(r v)}{\partial r}\right)\right)-\eta \frac{2 v}{r^{2}}-j_{z} B_{\theta}
\end{aligned}
$$

Conservation of Energy

in the arc region:

$$
\begin{aligned}
& \frac{\partial}{\partial z}(\rho h u)+\frac{1}{r} \frac{\partial}{\partial r}(\rho r h v) \\
& =\frac{\partial}{\partial z}\left(\frac{\lambda_{\mathrm{Ar}}}{C_{p_{\mathrm{Ar}}}} \frac{\partial h}{\partial z}\right)+\frac{1}{r} \frac{\partial}{\partial r}\left(\frac{\lambda_{\mathrm{Ar}}}{C_{p_{\mathrm{Ar}}}} r \frac{\partial h}{\partial r}\right)+\frac{j_{z}^{2}+j_{r}^{2}}{\sigma_{\mathrm{Ar}}} \\
& +\frac{5}{2} \frac{k_{\mathrm{b}}}{e}\left(\frac{j_{z}}{C_{p_{\mathrm{Ar}}}} \frac{\partial h}{\partial z}+\frac{j_{r}}{C_{p_{\mathrm{Ar}}}} \frac{\partial h}{\partial r}\right)-R a
\end{aligned}
$$

The radiation loss $R a$ from arc is given by the multipleterms approximation as a function of plasma temperature from the experimental data for argon. ${ }^{2)}$ inside the electrode

$$
\frac{\partial}{\partial z}\left(\frac{\lambda_{\mathrm{W}, \mathrm{Cu}}}{C_{p_{\mathrm{W}, \mathrm{Cu}}}} \frac{\partial h}{\partial z}\right)+\frac{1}{r} \frac{\partial}{\partial r}\left(\frac{\lambda_{\mathrm{W}, \mathrm{Cu}}}{C_{p_{\mathrm{W}, \mathrm{Cu}}}} r \frac{\partial h}{\partial r}\right)+\frac{j_{z}^{2}+j_{r}^{2}}{\sigma_{\mathrm{W}, \mathrm{Cu}}}=0 .
$$

Heat flux between arc and cathode, anode are respectively given by neglecting space charge effects ${ }^{13,14)}$ in the very thin sheath and both electrodes evaporation for simplicity ${ }^{15}$ )

$$
\begin{aligned}
& \dot{q}_{\mathrm{W} \leftrightarrow \mathrm{Ar}}=-\lambda_{\mathrm{W} \leftrightarrow \mathrm{Ar}}\left(\frac{\partial T}{\partial z}+\frac{\partial T}{\partial r}\right) \\
&-\left|j_{\mathrm{e}}\right|\left(\frac{5}{2} \frac{k_{\mathrm{b}} T_{\mathrm{Ar}}}{e}+\phi_{\mathrm{W}}\right)+\left|\mathbf{j}_{\mathrm{i}}\right|\left(V_{\mathrm{Ar}}+V_{\mathrm{w}}\right) \ldots \ldots . . \\
& \dot{q}_{\mathrm{Cu} \leftrightarrow \mathrm{Ar}}=-\lambda_{\mathrm{Cu} \leftrightarrow \mathrm{Ar}} \frac{\partial T}{\partial z}+j_{z}\left(\frac{5}{2} \frac{k_{\mathrm{b}} T_{\mathrm{Ar}}}{e}+V_{\mathrm{Cu}}+\phi_{\mathrm{Cu}}\right) \ldots
\end{aligned}
$$

where the emission current density $j_{\mathrm{e}}$ is given by the Richardson-Dushman equation

$$
j_{\mathrm{R}}=\left|j_{\mathrm{e}}\right|=A T_{\mathrm{s}}^{2} \exp \left(-\frac{e \phi_{\mathrm{W}}}{k_{\mathrm{b}} T_{\mathrm{s}}}\right) .
$$

ion current $\mathbf{j}_{\mathrm{i}}$ is given as follows:

$$
\left|\mathbf{j}_{\mathrm{i}}\right|=|\mathbf{j}|-j_{\mathrm{R}}\left(|\mathbf{j}|>j_{\mathrm{R}}\right), \quad\left|\mathbf{j}_{\mathrm{i}}\right|=0 \quad\left(|\mathbf{j}|<j_{\mathrm{R}}\right) \ldots
$$

where emission constant $A=1.2 \times 10^{6} \mathrm{~A} /\left(\mathrm{m}^{2} \mathrm{~K}^{2}\right)$, work function $\phi_{\mathrm{W}}=3.0 \mathrm{eV},{ }^{16)} \phi_{\mathrm{Cu}}=4.5 \mathrm{eV}^{13)}$ and ionization potential of argon $V_{\mathrm{Ar}}=15.8 \mathrm{eV}$.

Equation of State

$$
p=\rho R T
$$

Conservation of electric current

$$
\frac{\partial}{\partial z}\left(\sigma_{\mathrm{Ar}, \mathrm{W}, \mathrm{Cu}} \frac{\partial \phi}{\partial z}\right)+\frac{1}{r} \frac{\partial}{\partial r}\left(r \sigma_{\mathrm{Ar}, \mathrm{W}, \mathrm{Cu}} \frac{\partial \phi}{\partial r}\right)=0
$$

Ohmic equation

$$
\begin{aligned}
& j_{z}=-\sigma_{\mathrm{Ar}, \mathrm{W}, \mathrm{Cu}} \frac{\partial \phi}{\partial z} \\
& j_{r}=-\sigma_{\mathrm{Ar}, \mathrm{W}, \mathrm{Cu}} \frac{\partial \phi}{\partial r}
\end{aligned}
$$

Ampere's rule

$$
\frac{1}{r} \frac{\partial}{\partial r}\left(r B_{\theta}\right)=\mu_{0} j_{z}
$$

\subsection{Boundary Conditions}

In the previous almost studies, temperature distribution on the electrode surface and the current density at the cathode spot are given in assumptions. ${ }^{4-9)}$ In the present study, the temperature and electric potential conditions are given only at the cooled surfaces of anode and cathode, represented as lines $\mathrm{C}^{\prime} \mathrm{D}^{\prime}$, FA, in Fig. 1 without assuming the current density and temperature at the electrodes surface, represented as lines $\mathrm{CD}, \mathrm{FG}, \mathrm{GB}^{11)}$ and neglecting sheath boundary 
conditions for the reduction in computation time. ${ }^{15,17)}$ Because calculated arc temperature and cathode surface temperature are relatively insensitive to those detailed properties of the sheath. ${ }^{17,18)}$

axis $\mathrm{AB}: \quad \frac{\partial T}{\partial r}=0, \quad \frac{\partial \phi}{\partial r}=0$

axis $\mathrm{BC}: \quad \frac{\partial T}{\partial r}=0, \quad \frac{\partial \phi}{\partial r}=0, \quad \frac{\partial u}{\partial r}=v=0$

axis $\mathrm{CC}^{\prime}: \quad \frac{\partial T}{\partial r}=0, \quad \frac{\partial \phi}{\partial r}=0$

anode surface CD : $\quad u=v=0$

cooled anode surface $\mathrm{C}^{\prime} \mathrm{D}^{\prime}: \quad T=300 \mathrm{~K}, \phi=0$

anode side $\mathrm{DD}^{\prime}: \quad \frac{\partial^{2} T}{\partial r^{2}}=0, \quad \frac{\partial \phi}{\partial r}=0$

exit DE : $\frac{\partial^{2} T}{\partial r^{2}}=0, \quad \frac{\partial \phi}{\partial r}=0, \quad \frac{\partial u}{\partial r}=\frac{\partial v}{\partial r}=0$

exit $\mathrm{EF}^{\prime}: \quad \frac{\partial^{2} T}{\partial z^{2}}=0, \quad \frac{\partial \phi}{\partial z}=0, \quad \frac{\partial u}{\partial z}=\frac{\partial v}{\partial z}=0$

inlet $\mathrm{FF}^{\prime}: \quad \frac{\partial^{2} T}{\partial z^{2}}=0, \quad \frac{\partial \phi}{\partial z}=0, \quad u=u_{\text {in }}, \quad v=0$

cathode surface $\mathrm{FG}: \quad u=v=0$

cathode surface GB : $\quad u=v=0$

cooled cathode surface FA : $\quad T=300 \mathrm{~K}, \quad-\sigma_{\mathrm{W}} \frac{\partial \phi}{\partial z}=j_{\text {in }}$

\subsection{Numerical Conditions and Procedures}

Table 1 shows the discharge configuration and operating conditions referring to Gas Tungsten Arc (GTA) plasma facility. ${ }^{19)}$ A cylindrical thoriated tungsten cathode (W+ $2 \mathrm{wt} \% \mathrm{Th}$ ) and a plate copper anode are investigated for lifetime evaluation in the present study. The roots of both electrodes are cooled fixedly at $300 \mathrm{~K}$. The thermofluid field is solved by Semi-Implicit-Method for Pressure Linked Equation (SIMPLE) ${ }^{20)}$ method using Tri Diagonal-Matrix Algorithm (TDMA). The electromagnetic field is solved by Gauss-Seidel method. Here, the grid has $75 \times 102$ meshes in the axial and radial directions, respectively. The thermo-

Table 1. Discharge configuration and operating conditions.

\begin{tabular}{|c|c|}
\hline Operating pressure & $p_{0}=1.01 \times 10^{5} \quad(\mathrm{~Pa})$ \\
\hline Arc current & $I=50,150 \quad(\mathrm{~A})$ \\
\hline Gas flow rate & $Q=15,30 \quad(\mathrm{~S} \ell / \mathrm{min})$ \\
\hline Cathode radius & $r_{1}=1.6 \quad(\mathrm{~mm})$ \\
\hline Nozzle radius & $r_{2}=10.8 \quad(\mathrm{~mm})$ \\
\hline Anode radius & $r_{3}=25 \quad(\mathrm{~mm})$ \\
\hline Cathode vertex angle & $\theta=45^{\circ}, 60^{\circ}, 90^{\circ}$ \\
\hline Anode thickness & $z_{1}=5 \quad(\mathrm{~mm})$ \\
\hline Electrode gap & $z_{2}=5,10 \quad(\mathrm{~mm})$ \\
\hline $\begin{array}{l}\text { Computational } \\
\text { axial length }\end{array}$ & $z_{3}=22.8,27.8 \quad(\mathrm{~mm})$ \\
\hline
\end{tabular}

dynamic and transport properties of argon ${ }^{21,22)}$ and tungsten and copper ${ }^{23)}$ are given as a function of temperature.

\section{Cathode Lifetime Evaluation}

\subsection{Governing Equations}

Figure 2 shows a schematic illustration of a cylindrical thoriated tungsten cathode $(\mathrm{W}+2 \mathrm{wt} \% \mathrm{Th})$ and the coordinate system. Since radial diffusion plays a minor role and usually $L \gg r$, we use a simple one-dimensional (1D) model of the cathode, in which the spot entirely covers the hot side of cathode. It is important to clarify the thorium atom behavior in the cathode and that at the surface for the cathode lifetime evaluation. ${ }^{24,25)}$ Here the cathode lifetime is evaluated by coupling with plasma thermofluid characteristics as calculated previously. In order to derive the governing equations, the following assumptions are introduced.

(1) The analysis is one-dimensional (1D) in the $z$ axis for a cylindrical cathode with conical tip.

(2) There are constant emission current density and zero thorium concentration at the cathode spot of $z=0$.

(3) The thorium concentration gradient is always equal to zero in the constant cooling root of the cathode.

(4) The cathode length and cooling temperature are fixed but the tip vertex angle is variable.

(5) The cathode lifetime can be defined until when the surface temperature attains at the melting temperature.

To evaluate the cathode lifetime, the governing equation for thorium atom behavior ${ }^{16)}$ are presented as follows:

The diffusion in the cathode bulk is described by the Fick equation.

$$
\frac{\partial n}{\partial t}=\frac{\partial}{\partial z}\left(D(T) \frac{\partial n}{\partial z}\right)
$$

where diffusion coefficient

$$
D=D_{0} \exp \left(-\frac{e E}{k_{\mathrm{b}} T}\right)
$$

with $D_{0}=1.1 \times 10^{-4} \mathrm{~m}^{2} / \mathrm{s}$ and $E=4.1 \mathrm{eV}^{9)}$

The degree of coverage of thorium is governed by the balance equation

$$
N_{0} \frac{\partial \varphi}{\partial t}=-\Gamma_{\mathrm{eff}}\left(T_{\mathrm{s}}, \varphi\right)+\left.D\left(T_{\mathrm{s}}\right) \frac{\partial n}{\partial z}\right|_{z=0} \ldots
$$

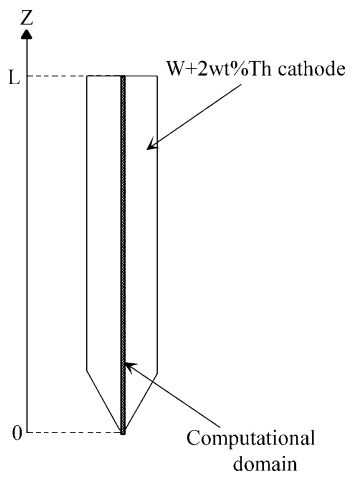

Fig. 2. Schematic illustration of a cylindrical thoriated tungsten cathode. 
which simply states that the variation in the number of thorium atoms on the cathode surface is equal to the number of atoms that arrive to the surface by diffusion from the cathode bulk minus the number of atoms lost by desorption. In Eq. (17) $N_{0}=4.2 \times 10^{18} \mathrm{~m}^{-2}$ 26) is the surface density of sites available for thorium atoms on the cathode surface. The last term on the right-hand side of Eq. (17) the diffusion flow of thorium atoms toward the spot.

The net desorption of thorium is described by

$$
\begin{gathered}
\Gamma_{\text {eff }}=U \cdot \xi \Gamma_{0} \exp \left(-\frac{e H}{k_{\mathrm{b}} T}\right) \ldots . \\
U(\varphi)=\left\{\begin{array}{l}
1: \varphi \geq 1 \\
\varphi: \varphi<1,
\end{array} \quad \xi=1.4 \times 10^{-2}\right.
\end{gathered}
$$

The heat of adsorption is $H(\varphi)=H_{\max }-\left(H_{\max }-H_{\min }\right) \varphi$ where the factor $\Gamma_{0}(T)=\left(k_{\mathrm{b}} T / h_{\text {plank }}\right) N_{0}$ corresponds to the desorption of thorium under vacuum. ${ }^{8)}$

The arc operates at a constant emission current density given by the Richardson-Dushman equation

$$
j_{\mathrm{R}}=\left|j_{\mathrm{e}}\right|=A T_{\mathrm{s}}^{2} \exp \left(-\frac{e \phi_{\mathrm{W}}}{k_{\mathrm{b}} T_{\mathrm{s}}}\right)
$$

where the experimental dependence of the effective work function with fractional coverage can be described by the simple expression.

$$
\begin{aligned}
& \phi_{\mathrm{w}}=\phi_{\min }+\left(\phi_{\max }-\phi_{\min }\right)(1-\varphi)^{2} \\
& \phi_{\min }=3.0 \mathrm{eV}, \quad \phi_{\max }=4.5 \mathrm{eV}
\end{aligned}
$$

Now $\left|j_{\mathrm{e}}\right|$ is given from the Eq. (8) in the arc analysis. The time dependent spatial distribution of temperature inside the cathode is given by energy equation.

$$
C_{p_{\mathrm{W}}}(T) \rho_{\mathrm{W}} \frac{\partial T}{\partial t}=\frac{\partial}{\partial z}\left(\lambda_{\mathrm{W}}(T) \frac{\partial T}{\partial z}\right)+\frac{j_{z}^{2}}{\sigma_{\mathrm{W}}}
$$

where specific heat, thermal conductivity and electrical conductivity are as a function of temperature.

Now $z$ component of current density $j_{z}(z)$ is given from energy equation of arc in Eq. (5).

\subsection{Initial Conditions and Boundary Conditions}

The initial conditions for Eqs. (15) and (21) are given by

$$
\begin{gathered}
\left\{\begin{array}{l}
n(0,0)=0 \\
n(z, 0)=n_{0}(z \neq 0)
\end{array}\right. \\
T\left(z, t_{0}\right)=\text { given }
\end{gathered}
$$

Here $T\left(z, t_{0}\right)$ is given from arc analysis.

On the other hand, the boundary conditions for Eqs. (15) and (21) are given by

$$
\begin{aligned}
& \left\{\begin{array}{l}
n(0, t)=0 \\
n(L, t)=n_{0}=8.8 \times 10^{26} \mathrm{~m}^{-3}
\end{array}\right. \\
& \left\{\begin{array}{l}
T(0, t)=T_{\mathrm{s}}(t) \\
T(L, t)=T_{0}=300 \mathrm{~K}
\end{array}\right.
\end{aligned}
$$
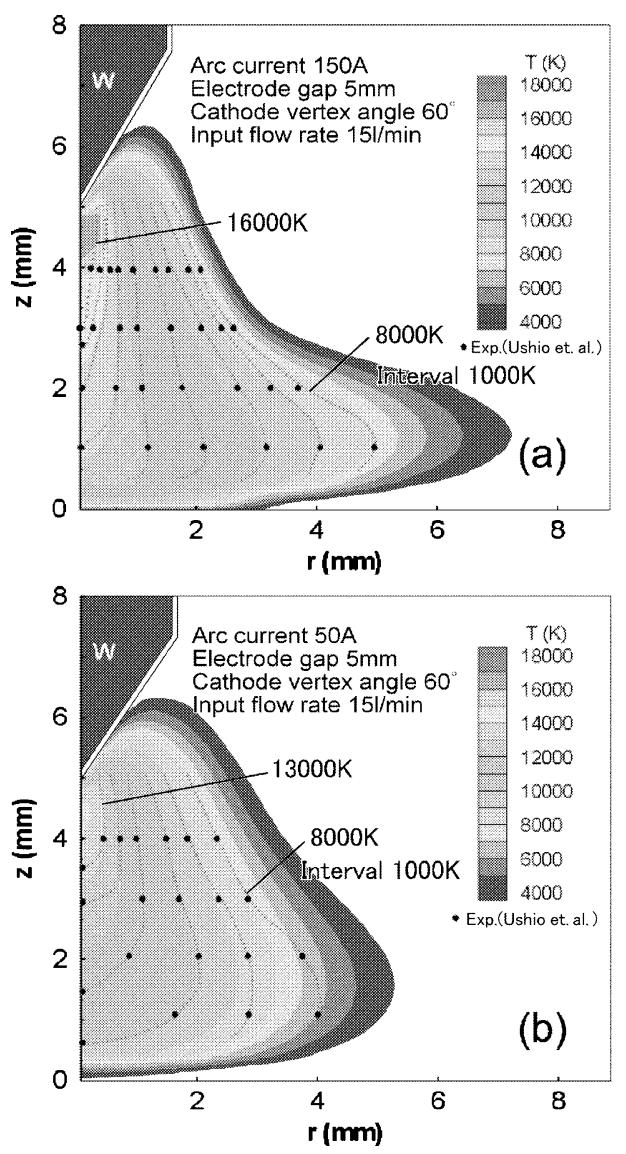

Fig. 3. Effect of arc current on plasma temperature. ((a) in the case of arc current $150 \mathrm{~A}$, (b) $50 \mathrm{~A}$ )

Here $T_{\mathrm{s}}(t)$ is obtained from Eq. (19).

Each governing equation are descretized by the control volume method. The number of grids is 101 and time step is $0.01 \mathrm{~s}$.

\section{Numerical Results and Discussion}

Figures 3(a) and 3(b) show the effect of arc current on the plasma temperatures with the available experimental data. ${ }^{19)}$ The reference condition is arc current $150 \mathrm{~A}$, electrode gap $5 \mathrm{~mm}$, cathode vertex angle $60^{\circ}$ and inlet flow rate $15 \mathrm{l} / \mathrm{min}$ for parametric comparisons later. There is maximum temperature near cathode tip with large electric current and also showing bell configuration with impinging jet. When the arc current increases from 50 to $150 \mathrm{~A}$, the maximum temperature near the cathode tip increases more than $16000 \mathrm{~K}$ and high temperature region elongates toward the anode due to the active Joule heating. Furthermore, it spreads along the anode surface due to the accelerated impinging velocity by the stronger pinch effect.

There is a good quantitative agreement with the experimental data in a whole region under the LTE condition. However, there is some discrepancy near the cathode tip at $150 \mathrm{~A}$ and near the arc fringe at $50 \mathrm{~A}$ respectively. This thermal nonequilibrium effect comes from the much inflowing of colder gas into arc core at $150 \mathrm{~A}$ and also insufficient collision between electrons and heavy particles near the fringe at $50 \mathrm{~A} .{ }^{19)}$

Figures 4(a) and 4(b) show the effect of arc current on the axial velocities. When the arc current increases from 50 

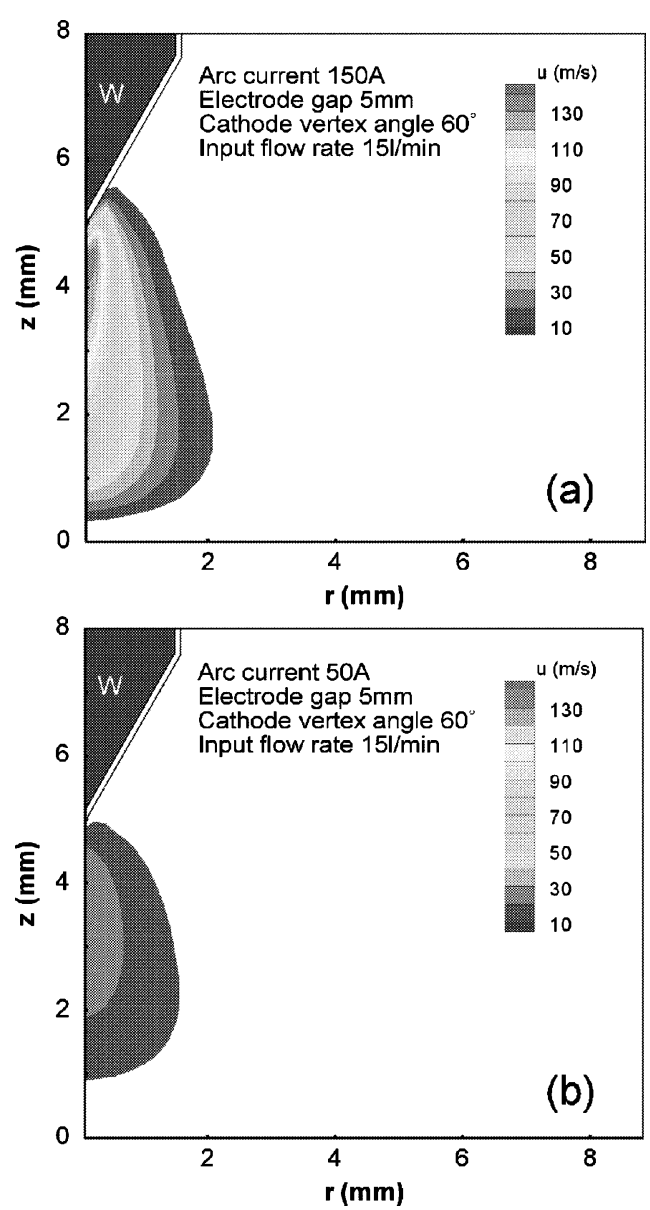

Fig. 4. Effect of arc current on plasma velocity. ((a) in the case of arc current $150 \mathrm{~A}$, (b) $50 \mathrm{~A}$ )

to $150 \mathrm{~A}$, the maximum velocity near cathode tip increases considerably from 27 to $138 \mathrm{~m} / \mathrm{s}$. This results from the acceleration of axial velocity by increasing in the radial and axial Lorenz forces at $150 \mathrm{~A}$. Therefore, the arc spreads along the anode surface with stronger impinging velocity.

Figures 5(a) and 5(b) show the effect of arc current on the electric current density. It is clearly shown that the electric current attains maximum near the cathode tip due to the steep gradient of electric potential at $150 \mathrm{~A}$.

Figure 6 shows the plasma temperature at the electrode gap of $10 \mathrm{~mm}$. When the electrode gap increases, the whole plasma temperature decreases compared with that as shown in Fig. 3(a) due to the large heat loss in the radial direction from larger arc surface.

Figure 7 shows the plasma temperature for inlet flow rate of $30 \mathrm{l} / \mathrm{min}$. There is little variation of whole temperature field in the free burning area in the present study, but showing a relative elongation toward the anode compared with that as shown in Fig. 3(a).

Figures 8(a) and 8(b) show the plasma temperatures for cathode vertex angles of $45^{\circ}$ and $90^{\circ}$ respectively. The high temperature region up to $18000 \mathrm{~K}$ is observed in the core region near cathode tip of $45^{\circ}$ due to the active Joule heating production induced by the electric current concentrations. On the other hand, the high temperature region at $90^{\circ}$ becomes considerably smaller near cathode tip and along the anode compared with that at $60^{\circ}$ as shown in Fig. 3(a), without electric current concentration so much.
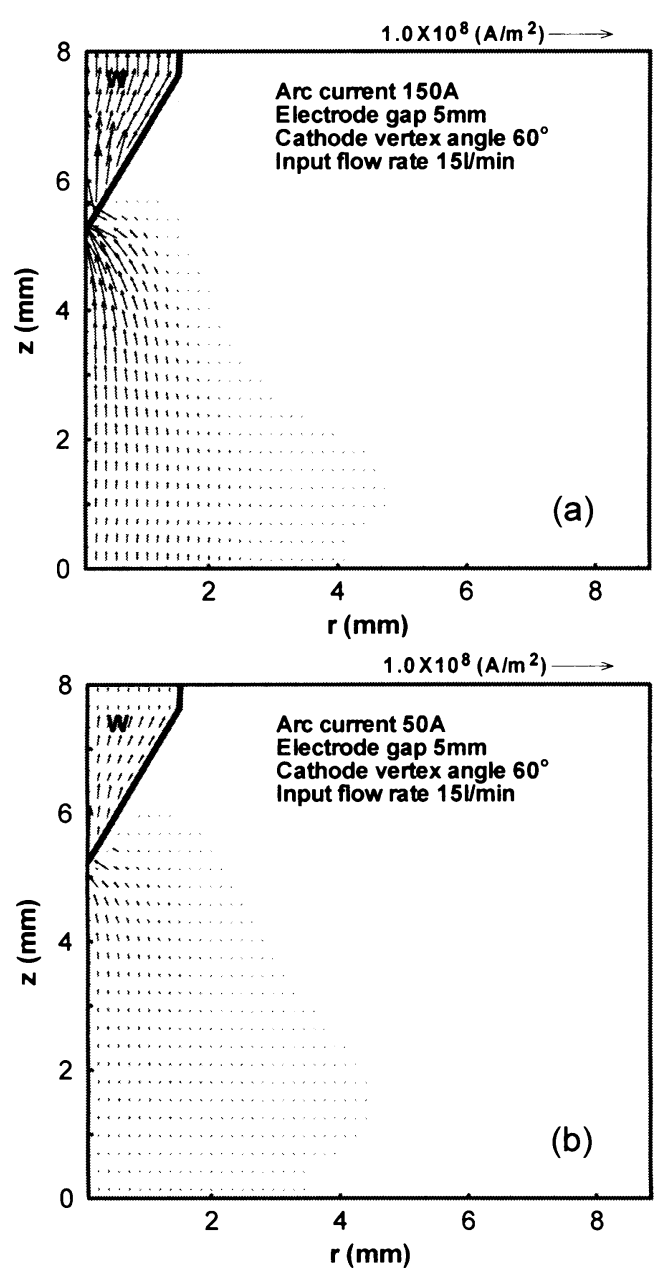

Fig. 5. Effect of arc current on electric current density. ((a) in the case of arc current $150 \mathrm{~A}$, (b) $50 \mathrm{~A}$ )

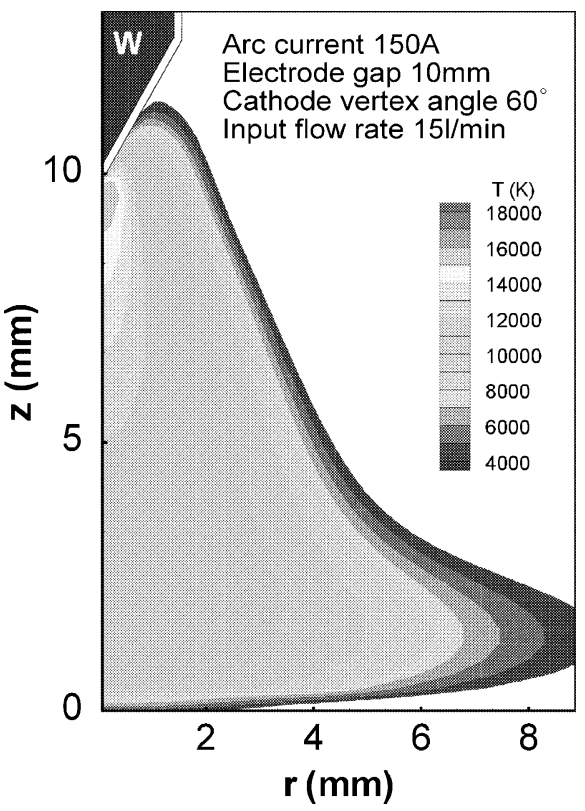

Fig. 6. Effect of electrode gap on plasma temperature.

Figure 9 shows the time evolution of cathode temperature for $150 \mathrm{~A}$ and $60^{\circ}$. The cathode temperature shows the moderate variation from the water cooled root to the cathode tip for $130 \mathrm{~h}$. Over $135 \mathrm{~h}$, the temperature near the cathode tip suddenly attains the melting point of $3693 \mathrm{~K}$ ac- 


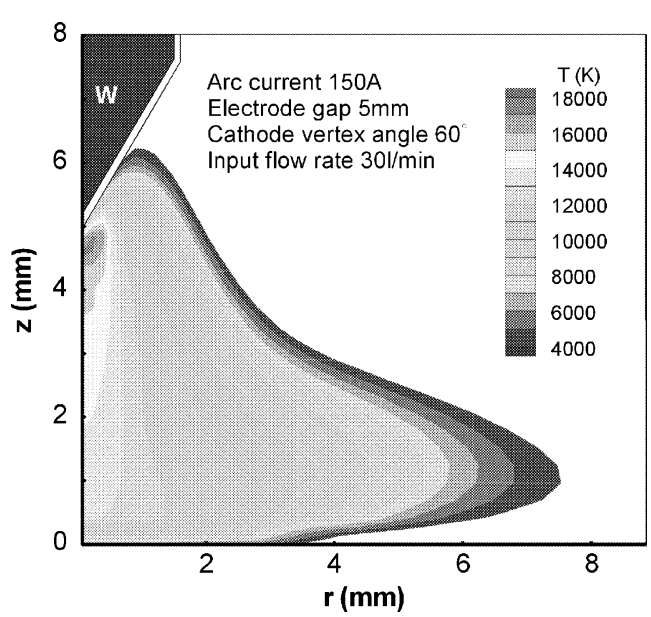

Fig. 7. Effect of inlet flow rate on plasma temperature.
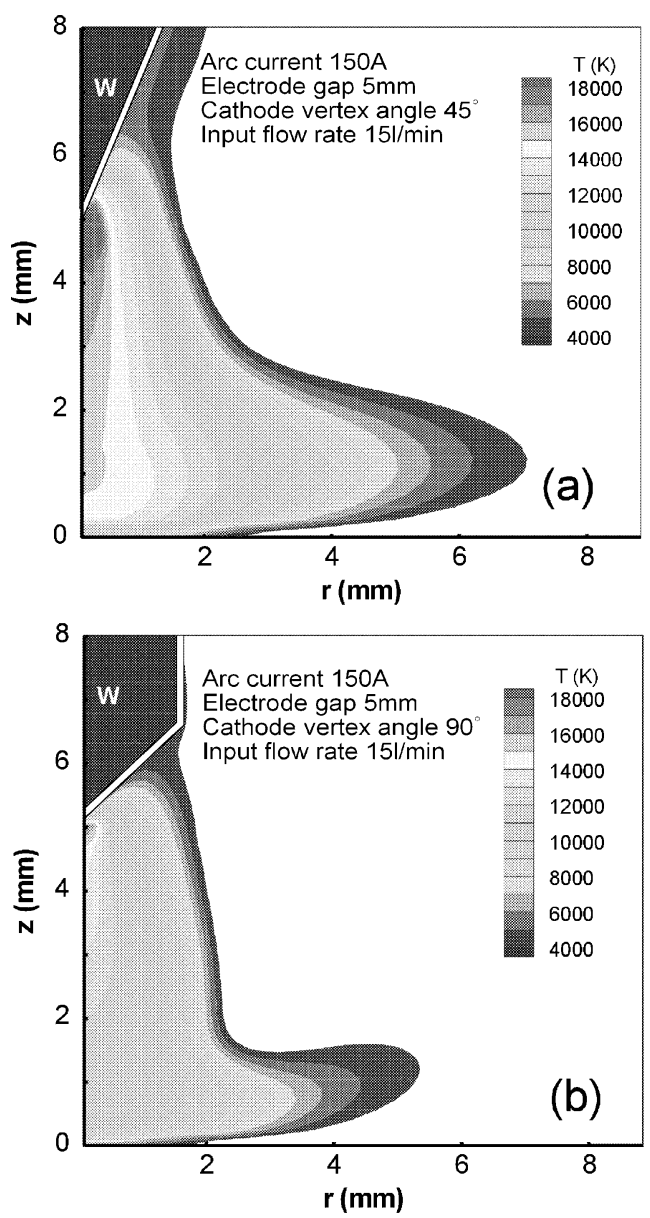

Fig. 8. Effect of cathode vertex angle on plasma temperature. ((a) in the case of cathode vertex angle $45^{\circ}$, (b) $90^{\circ}$ )

companied by melting errosion, which means the lifetime of electrode.

Figure 10 shows the effects of the arc current and cathode vertex angle on the cathode lifetime. Corresponding to Fig. 9, cathode tip temperature shows nearly constant for a while and then rapidly increases to the melting point. When the arc current changes form 50 to $150 \mathrm{~A}$, the lifetime of electrode becomes less than $50 \%$.

The lifetime for $45^{\circ}$ tip vertex is the shortest, but that for $60^{\circ}$ tip vertex is the longest. Since the work function increases relatively due to the increase in the desorption of

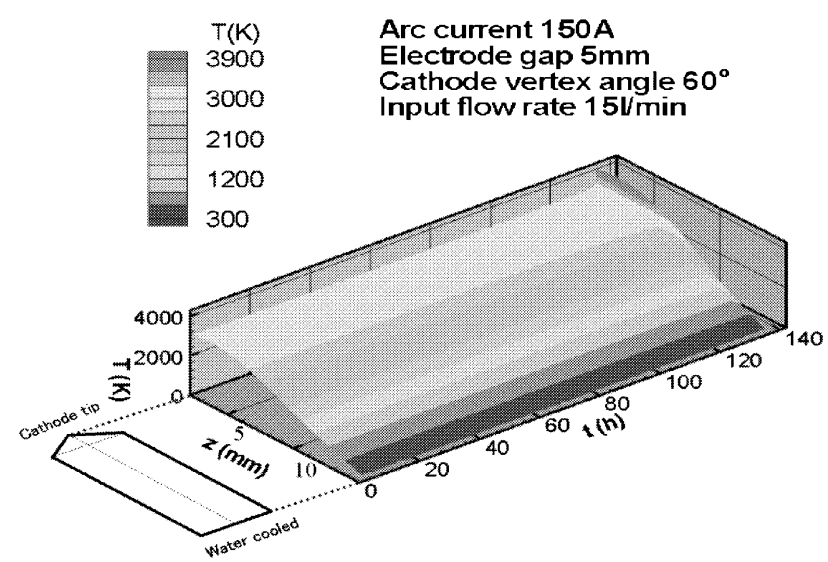

Fig. 9. Evolution of cathode temperature with time.

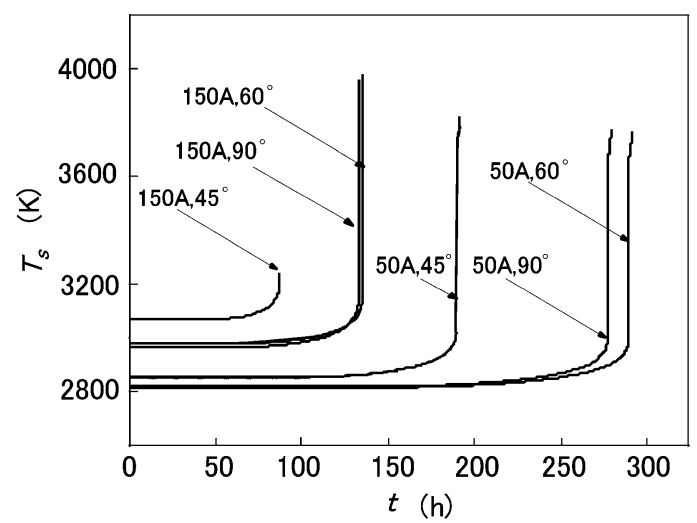

Fig. 10. Effects of arc current and cathode vertex angle on cathode lifetime.

thorium induced by electric current concentration, the tip temperature increases drastically at $45^{\circ}$ following Eq. (8). When the tip vertex angles change from 60 to $90^{\circ}$, however, the lifetime decreases a little. These result from that the thorium can not be sufficiently diffused toward the cathode tip due to the decrease in the Joule heating inside the cathode, although there is decrease in the adsorption of thorium from cathode tip induced by decrease in the electric current.

\section{Concluding Remarks}

The thermofluid analysis of a free burning arc is conducted considering the arc-electrodes interactions without empirical assumptions. Subsequently, the cathode lifetime is evaluated by coupling with arc analysis for optimization. The results obtained by computational simulation are as follows:

(1) The arc current, which is an important parameter for plasma temperature and velocity fields, increases the maximum temperature near the cathode tip due to the active Joule heating and increases the axial velocity due to the strong pinch effect.

(2) The highest temperature region is observed at the cathode tip vertex angle of $45^{\circ}$ due to the strong electric current concentration.

(3) There are small effects of the electrode gap and inflow rate on the thermofluid field.

(4) There is a good quantitative agreement of plasma temperature with experimental data under the LTE condi- 
tion, except near cathode tip and arc fringe.

(5) The cathode lifetime decreases considerably with increase in the arc current. It is the shortest for cathode vertex angle of $45^{\circ}$ and there exists the optimum vertex angle near $60^{\circ}$ for the longest lifetime.

\section{Acknowledgements}

We would like to give our sincere thanks to Professor Masao Ushio and Dr. Manabu Tanaka at Welding and Jointing Institute, Osaka University for their valuable discussions and important materials. The present work was partly supported by Grant-in-aid for Scientific Research (B) from Japan Society of Promotion Science (2001). The numerical simulation was conducted by Origin2000 at Advanced Fluid Information Research Center, Institute of Fluid Science, Tohoku University.

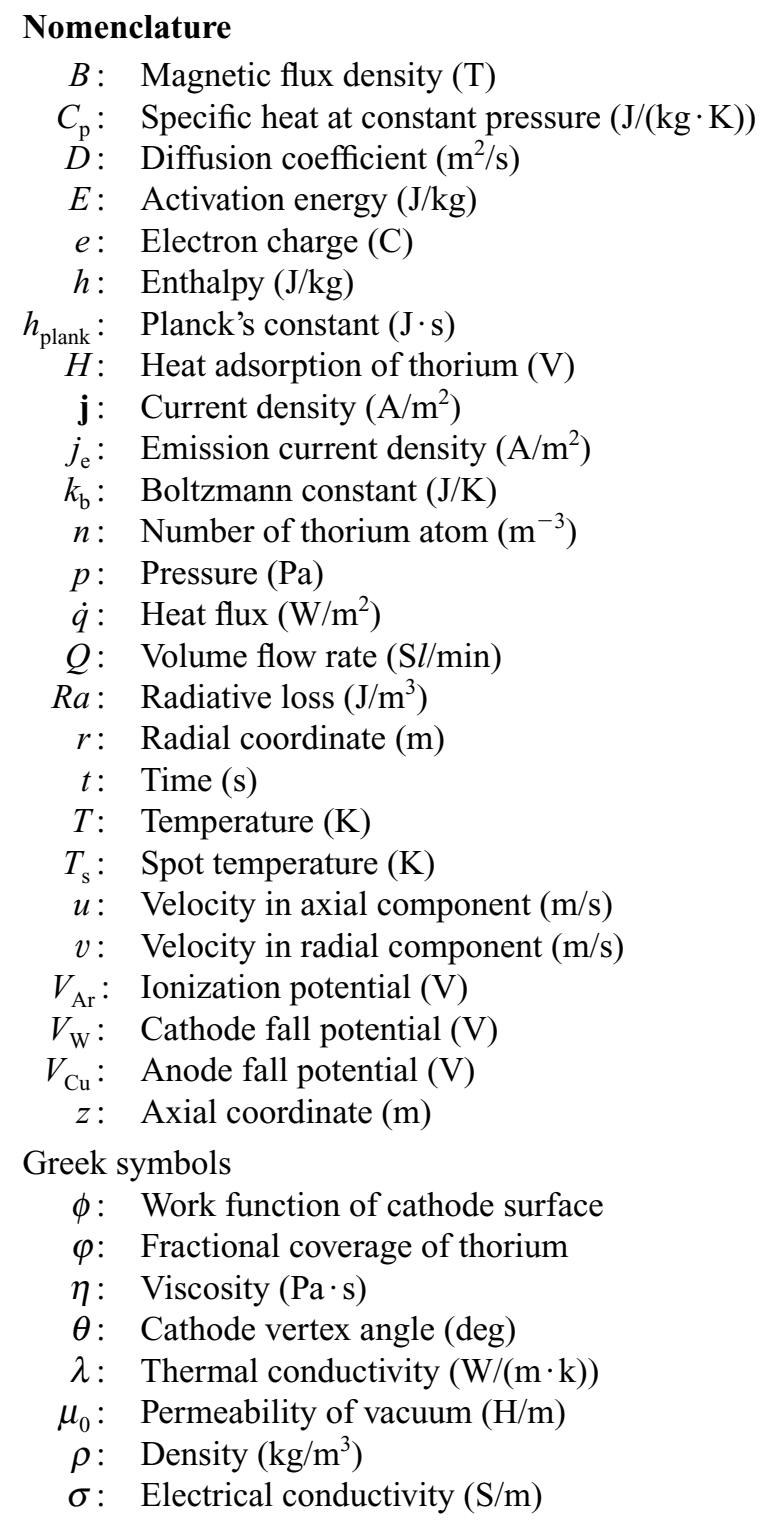

$$
\Gamma: \text { Desorption of thorium }\left(\mathrm{m}^{2} / \mathrm{s}\right)
$$

Subscripts

$\begin{aligned} & \text { Ar: } \text { Argon } \\ & \mathrm{W}: \text { Tungsten } \\ & \mathrm{Cu}: \text { Copper } \\ & \mathrm{e}: \text { Electron } \\ & \mathrm{i}: \text { Ion } \\ & r: \text { Radial component } \\ & \mathrm{s}: \text { Cathode tip } \\ & z: \text { Axial component } \\ & \theta: \text { Azimuthal component }\end{aligned}$

\section{REFERENCES}

1) Functional Fluids and Intelligent Fluids, ed. by Japan Society of Mechanical Engineers, Corona Public Corp. Ltd., Tokyo, (2000), 48.

2) T. Iwao, H. Miyazaki, T. Ishida, Y. Liu and T. Inaba: ISIJ Int., 40 (2000), 275.

3) E. Pfender: Plasma Chem. Plasma Process., 19 (1996), 21.

4) K. C. Hsu, K. Etemadi and E. Pfender: J. Appl. Phys., 54 (1983), 1293.

5) A. B. Murphy: Phys. Rev. E, 55 (1997), 7473.

6) J. Menart, S. Malik and L. Liu: J. Phys. D: Appl. Phys., 33 (2000), 257.

7) R. C. Bianchini, R. C. Favalli, M. M. Pimenta and R. N. Szente: J. Phys. D: Appl. Phys., 33 (2000), 134.

8) E. L. Belousova: Sov. Phys. Tech. Phys., 25 (1980), 471.

9) P. Solana, P. Kapadia and J. Dowden: J. Phys. D: Appl. Phys., 31 (1998), 3446.

10) X. Chen and H.-P. Li: Int. J. Heat Mass Transfer., 44 (2001), 2541.

11) H. Nishiyama, T. Sato and K. Takamura: Proc. of Fluids Eng. Conf., JSME, Tokyo, (2001), CD-ROM 518.

12) D. L. Evans and R. S. Tankin: Phys. Fluids, 10 (1967), 1137.

13) N. A. Sanders and E. Pfender: J. Appl. Phys., 55 (1984), 714.

14) A. Kanzawa: Plasma Heat Transfer, Shinzansha-Sytech Corp., Tokyo, (1992).

15) J. J. Lowke, P. Kovitya and H. P. Schmidt: J. Phys. D: Appl. Phys., 25 (1992), 1600.

16) E. Casado and V. Colomer: J. Phys. D: Appl. Phys., 33 (2000), 1342.

17) P. Zhu, J. J. Lowke and R. Morrow: J. Phys. D: Appl. Phys., 25 (1992), 1369.

18) J. J. Lowke, R. Morrow and J. Haidar: J. Phys. D: Appl. Phys., 30 (1997), 2033.

19) M. Ushio, M. Tanaka and T. Sakaue: Proc. of the Spring Meeting, Japan Welding Society, 58 (1996), 72.

20) S. V. Patankar: Numerical Heat Transfer and Fluid Flow, Hemisphere Pub. Corp., New York, (1980).

21) M. I. Boulos, P. Fauchais and E. Pfender: Thermal Plasmas: Fundamentals and Applications Vol.1, Plenum Press, New York, (1994), 388 .

22) J. Menart and L. Lin: J. Thermophys. Heat Transf., 12 (1998), 500.

23) Hand Book of Chemistry, Fundamental II, ed. by Japan Chemical Engineering of Society, Maruzen, Tokyo, (1975), 985.

24) J. Haidar and A. J. D. Farmer: J. Phys. D: Appl. Phys., 28 (1995), 2089.

$25)$ B. Pokrzywka, S. Pellerin, K. Musiol, F. Richard and J. Chapelle: $J$. Phys. D: Appl. Phys., 29 (1996), 2841.

26) J. P. Estrup, J. Anderson and W. E. Danforth: Surf. Sci., 4 (1966), 286. 